\title{
Human Tooth Culture: A Study Model for Reparative Dentinogenesis and Direct Pulp Capping Materials Biocompatibility
}

\author{
Odile Téclès, Patrick Laurent, Virginie Aubut, Imad About \\ Laboratoire IMEB-ERT 30, Faculté d'Odontologie, Université de la Méditerranée, 27 Boulevard Jean Moulin, \\ 13355 Marseille Cedex 05, France
}

\begin{abstract}
In a previous work, based on an in vitro entire tooth culture model of human immature third molars, we demonstrated that perivascular progenitor cells can proliferate and migrate to the injury site after pulp exposure. In this work, we investigated the differentiation of cells after direct capping with biomaterials classically used in restorative dentistry. Histological staining after direct pulp capping with Calcium Hydroxide $X R^{\mathbb{R}}$ or MTA revealed early and progressive mineralized foci formation containing BrdU-labeled sequestered cells. The molecular characterization of the matrix and the sequestered cells by immunohistochemistry (Collagene type I, Dentin sialoprotein, and Nestin) clearly demonstrates that these areas share common characteristics of the mineralized matrix of reparative dentin formed by odontoblast-like cells. This reproduces some features of the pulp responses after applying these materials in vivo and demonstrates that the entire tooth culture model reproduces a part of the early steps of dentin regeneration in vivo. Its future development may be useful in studying the effects of biomaterials on this process. (C) 2007 Wiley Periodicals, Inc. J Biomed Mater Res Part B: Appl Biomater 85B: 180-187, 2008
\end{abstract}

Keywords: entire tooth culture; osteodentin; odontoblast; differentiation; reparative dentinogenesis

\section{INTRODUCTION}

During tooth development, inductive epithelial-mesenchymal interactions lead to the differentiation of ectomesenchymal pulp cells into odontoblasts, highly differentiated postmitotic cells. ${ }^{1}$

In pathological conditions such as moderate carious lesions or cavity preparation, the odontoblasts have the ability to respond to injury and upregulate their secretory activity leading to the deposition of reactionary dentin. ${ }^{2,3}$ When the intensity of the injury leads to odontoblast death, a new generation of odontoblast-like cells replace the necrotic cells and secrete a reparative dentin matrix that isolates the underlying healthy pulp from the exposure site. ${ }^{4}$ In this case, the lesions are clinically treated by applying a pulp capping material such as calcium hydroxide $\mathrm{XR}^{\circledR}$ or Mineral Trioxide Aggregate (Pro Root MTA $^{\circledR}$ ) directly onto the pulp core (direct pulp capping).
Several studies have suggested the presence of progenitor stem cells in the pulp and several origins of these cells were suggested. These include the sub-odontoblast cells in the layer of Höhl, fibroblasts, undifferentiated mesenchymal cells from the pulp core, and vascular-derived pericytes. ${ }^{2}$ The pulp progenitor stem cells have been shown to be multipotent. It has been shown that the adult human dental pulp contains a rapidly proliferative subpopulation of cells capable of differentiating into adipocytes and neural-like cells. ${ }^{5}$ In vitro, they can differentiate into odontoblast-like cells secreting a mineralized matrix having the mineral and molecular characteristics of dentin. ${ }^{6}$ We have recently developed a new human entire tooth culture model to study the early steps of dentin regeneration. In case of pulp exposure, this model showed that perivascular pulp progenitor cells proliferate in response to odontoblast injury and migrate to the pulpal injury site whereas teeth cultured with dentin cavities did not show any perivascular proliferation. ${ }^{7}$ Thus, reproducing the major difference of pulp response to dentin cavities and pulp exposure in vivo. However, it is still unclear if the migrating cells in this model are able to differentiate into odontoblast-like cells.

The purpose of this study was to find out if cells are able to differentiate and secrete a mineralized matrix in 
this entire tooth culture model and to find out if the obtained pulp response corresponds to that observed in vivo after restoration with classically used direct pulp capping materials.

\section{MATERIALS AND METHODS}

\section{Materials}

For the preparation of the culture media, all materials were purchased from Cambrex (Cambrex Bio Science, Walkersville, MD). Chemicals were obtained from Sigma-Aldrich (Sigma Aldrich Chemicals Corp, St. Louis, MO) unless otherwise precised.

\section{Teeth}

Thirty-five unimpacted immature human third molars freshly extracted for orthodontic reasons were obtained from 15- to 18-year-old adolescents in compliance with French legislation (informed patients and parents consent and Institutional Review Board approval of the protocol used). The teeth were used to study the differentiation of pulp progenitor cells after preparation of pulpal cavities. Only teeth showing one to two third root formations were used.

\section{Antibodies}

Polyclonal antibodies against the type I collagen were purchased from Southern Biotechnology Associates. (Birmingham, AL). Polyclonal antibodies against dentin-sialoprotein were given by Dr. Butler (University of Texas, USA). Monoclonal antibodies against nestin were purchased from Chemicon (Chemicon International, CA) and those against BrdU were from Sigma-Aldrich (Sigma Aldrich Chemicals Corp, St. Louis, MO).

\section{Tooth Culture}

The extracted teeth were immediately transported to the laboratory in minimum essential medium (MEM) supplemented with $300 \mathrm{UI} / \mathrm{mL}$ penicillin, $300 \mu \mathrm{g} / \mathrm{mL}$ streptomycin, and $0.75 \mu \mathrm{g} / \mathrm{mL}$ amphotericin $\mathrm{B}$ (fungizone ${ }^{\circledR}$ ). The teeth were cleaned with sterilized instruments after eliminating the periodontal ligament and the dental sac. Exposed pulpal cavities were prepared with diamond truncated burs (reference 878.2872) mounted on a high-speed $(300,000$ rpm) dental hand-piece and sterile saline cooling. The bur was left at speed until pulp penetration then it was retracted. This penetration was reached upon the disappearance of the hard tissue resistance. The teeth were restored with classically used pulp capping materials on a random basis: Mineral Trioxide Aggregate (Pro Root MTA ${ }^{\circledR}$ ) or calcium hydroxide $\mathrm{XR}^{\circledR}$ according to the manufacturers' instructions. For BrdU-labeling, the teeth were incubated with BrdU-containing medium $(1 \mathrm{mg} / \mathrm{mL})$ for 1 day. Then, they were cultured in the same medium without BrdU for 1,14 , or 28 days ( $n=5$ per group and per material). A control group was restored with agar-containing MEM medium and cultured under the same conditions $(n=5)$. The teeth were cultured in 12-well tissue plates (Falcon ${ }^{\circledR}$, Becton Dickinson, USA) in Minimum Essential Medium (MEM) supplemented with $10 \%$ fetal bovine serum and 2 $\mathrm{m} M \beta$-glycerophosphate. The crowns were fixed to a metallic wire with a sealant (Embrace ${ }^{\circledR}$, Pulpdent, USA). The wire was suspended on the two adjacent plates. The apical part of the teeth was dipped in $4 \mathrm{~mL}$ of culture medium without touching the bottom of the culture wells in order to facilitate the diffusion of the medium through the apical part without any direct contact with the restorative material (Figure 1). The culture medium was changed every day.

\section{Histology}

At the end of the culture periods, the teeth were fixed in $4 \%$ formol solution and routinely processed on longitudinal sections as described previously. ${ }^{7}$

\section{Scanning Electron Microscopy}

Scanning electron microscopy (SEM) examination was performed to examine the details of the pulpal mineralization on deparaffinated and rehydrated $7 \mu$ m-thick sections. Samples both from MTA and calcium hydroxide were sputtercoated with gold (JEOL, JCS 1100, Tokyo, Japan) and examined using a FEI type QUANTA 200 (FEI, France, LIMEIL BREVANNES, France).

\section{Immunohistochemistry}

To investigate the cell differentiation, odontoblast molecular markers were examined. This was performed on rehydrated paraffin embedded sections with antibodies against collagen I, dentin sialoprotein, and nestin. Primary antibodies were diluted in PBS containing $0.1 \%$ bovine serum albumin (BSA). The incubation with primary antibodies was performed overnight at $4{ }^{\circ} \mathrm{C}$ at the following concentrations: anti-BrdU antibodies were used at $10 \mu \mathrm{g} / \mathrm{mL}$, anticollagen I antibodies at $40 \mu \mathrm{g} / \mathrm{mL}$, and anti-nestin antibodies at $5 \mu \mathrm{g} /$ $\mathrm{mL}$. Anti-dentinsialoprotein antibodies were diluted 1:100 in PBS. The staining was revealed using the labeled streptavidin-biotin kit (LSAB) (DakoCytomation, Carpinteria, CA) according to the manufacturer's instructions. Controls were performed by incubations with unrelated primary antibodies. All controls gave negative results.

\section{Mineralization Morphometric Measurements}

These were performed with an Image Processing System IPS 32 version 4.32 (Samba technologies, Grenoble, France). The measurements were done on three representative hematoxylin-eosin stained sections per tooth.

Image analysis was used to quantify the foci surface, their mean distance from the exposure and the pulp cham- 

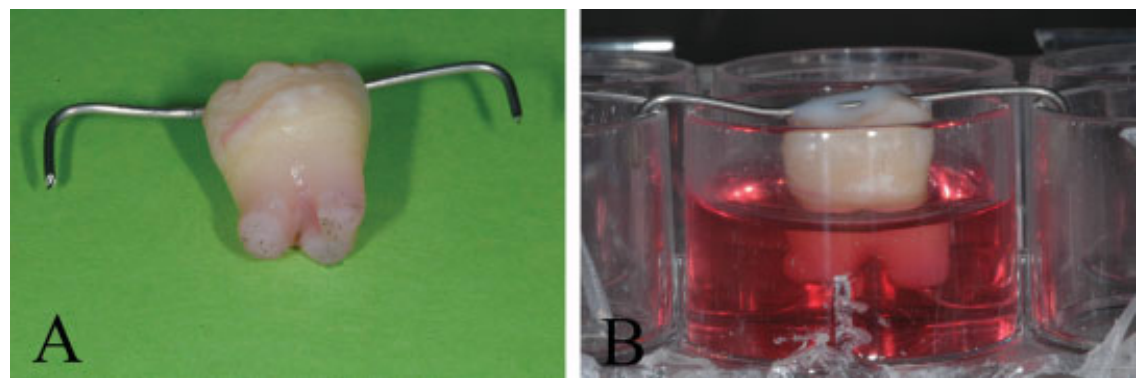

Figure 1. Entire tooth culture system. After cavities preparations and restorations, the teeth were fixed by the crowns to a wire $(A)$ and were suspended in the culture medium in 12-well tissue plates $(B)$, thus permitting the diffusion of the medium through the apex without any direct contact between the biomaterial and the medium. [Color figure can be viewed in the online issue, which is available at www.interscience.wiley.com.]

ber surface. The pulp surface mineralization percentage was calculated from the foci total surface and the pulp chamber area.

\section{Statistical Analysis}

A two-way analysis of variance was performed (material and culture period) followed by a Duncan test. The significance level was set at 0.05 .

\section{RESULTS}

\section{Histology}

The microscopic analysis showed major histological changes in the reaction zone close to the material-pulp interface. The rest of the underlying pulp tissue was consistently found to be of normal morphology without evident signs of tissue degeneration. In control teeth, no modification was observed except a fibrous condensation just beneath the cavity (not shown).

After MTA application and culture for 1 day, a thin acellular necrotic zone was located at the capping materialpulpal tissue interface and was clearly distinguishable from the rest of the pulp tissue [Figure 2(A,B)]. A denser connective tissue area under this homogenous zone and dilated venules were common findings [Figure 2(B)]. At this stage, some small and dense foci appeared within this zone and the adjacent pulp tissue [Figure 2(A,C)]. Cells were enclosed in these spherical zones which took the appearance of osteodentin. After culture periods of 14 and 28 days, more foci were observed. These foci surrounded the injured pulp tissue were located beneath the restorative material [Figure 3(A-C)]. The sequestered cells were BrdU-labeled [Figure 3(D)].

\section{Immunohistochemistry}

The mineralized foci molecular characterization [Figure $3(\mathrm{E}-\mathrm{H})]$ revealed that type I collagen immunoreactivity was strong and uniform in pulpal matrix and in the fibroblasts. This result was independent of the culture period and the material used. The expression of collagen I was evident in the mineralized foci matrix and its intensity was similar to dentin. A strong labeling was also observed in the sequestered cells [Figure 3(E)].

Nestin expression was localized in the foci and the intensity was strong in the sequestered cells [Figure 3(F,H)]. Dentin sialoprotein immunolabeling was observed in the pulp matrix and in the sequestered cells and the labeling intensity was similar in both localizations. In some foci, the
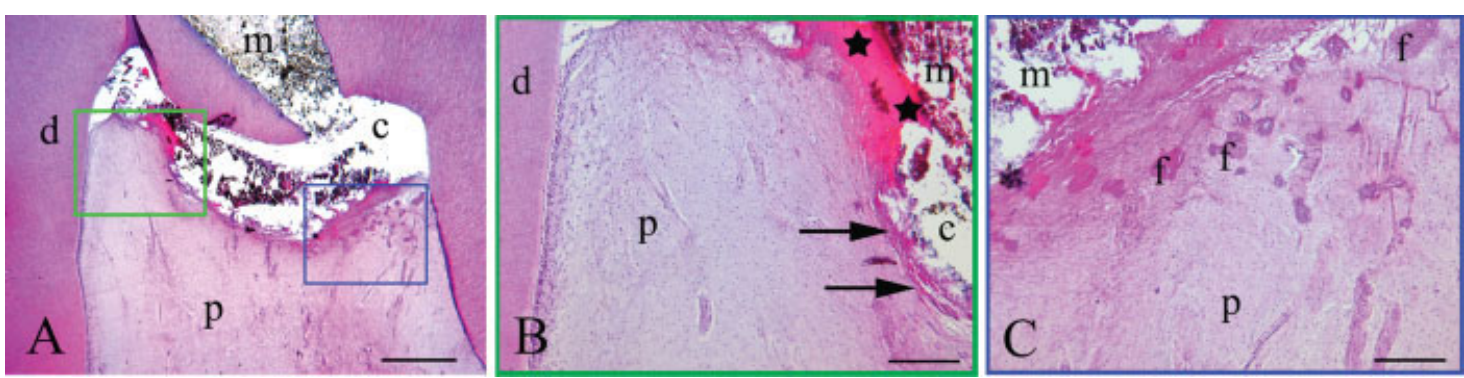

Figure 2. A view of pulp cusp area of a tooth capped with MTA and cultured for1 day. A thin acellular necrotic zone (stars) was located at the capping material-pulpal tissue interface (A, B). A condensed layer of connective tissue (arrows) is seen at the border of the wound surface (B). A particular zone near the exposure site shows mineralized foci $(A, C)$. H\&E staining. $d$, dentin; $p$, pulp; c, cavity; m, biomaterial; f, foci. Scale bars: $A=500 \mu \mathrm{m}$; B and $\mathrm{C}=200 \mu \mathrm{m}$. [Color figure can be viewed in the online issue, which is available at www.interscience.wiley.com.] 

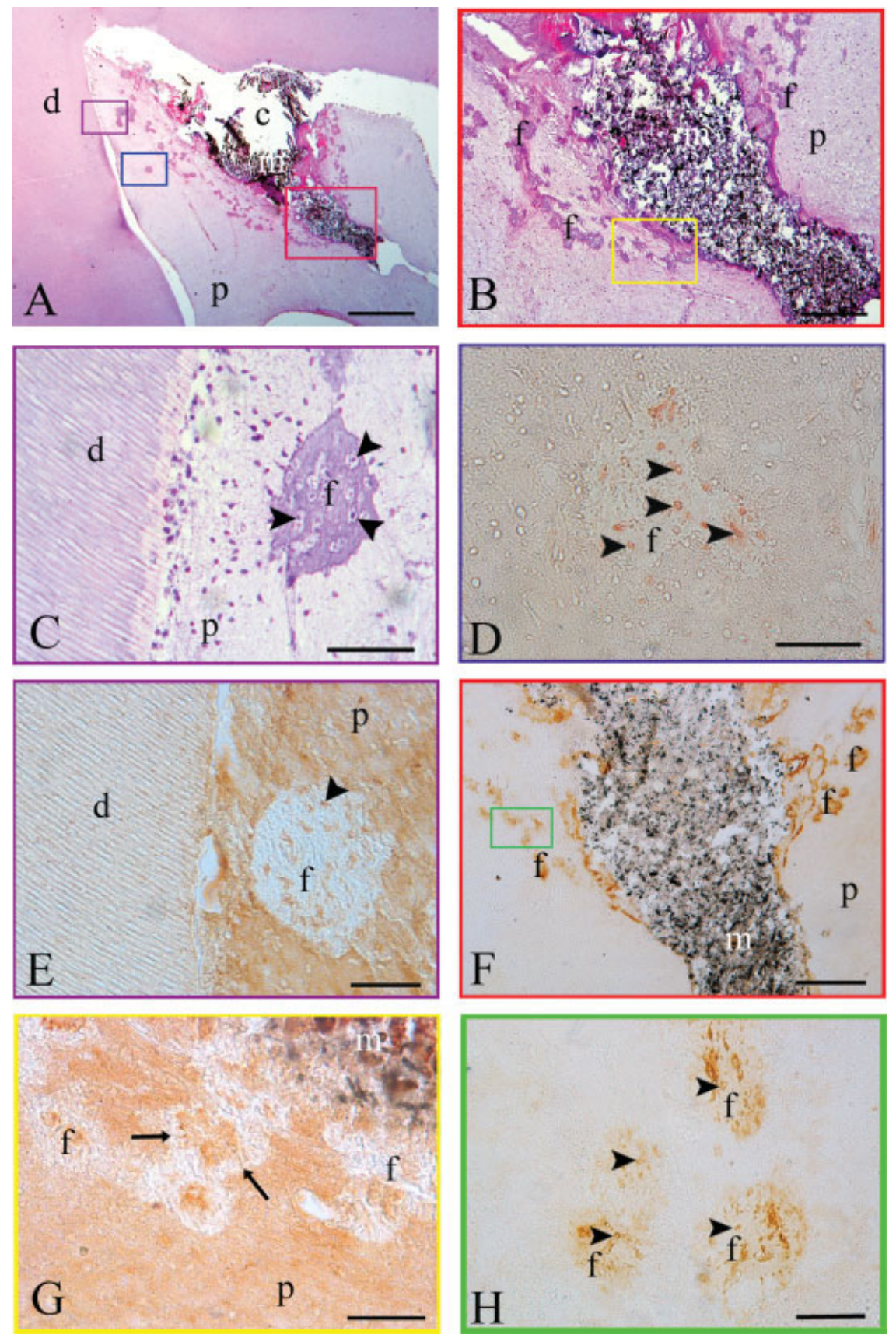

Figure 3. A view of cusp area of a tooth capped with MTA and cultured for 2 weeks after the BrdU labelling. Mineralized foci are observed in the vicinity of the material (A, B). These mineralized foci contain sequestered cells (arrowheads) (C). These sequestered cells are BrdU-labeled (arrowheads) (D). Collagen I immunostaining intensity is weak in the dentin and in the foci matrix but strong in pulpal tissue and sequestered cells $(E)$. Dentin sialoprotein is observed in the pulp tissue and in the sequestered cells $(G)$. Nestin immunolabelling is localized in the mineralized foci sequestered cells (F, H). A-C: H\&E staining. d, dentin; $p$, pulp; c, cavity; $m$, biomaterial; $f$, foci. Scale bars: $A=500$ $\mu \mathrm{m} ; \mathrm{B}$ and $\mathrm{F}=200 \mu \mathrm{m} ; \mathrm{C}-\mathrm{E}, \mathrm{G}$, and $\mathrm{H}=50 \mu \mathrm{m}$. [Color figure can be viewed in the online issue, which is available at www.interscience.wiley.com.]

sequestered cells exhibited tubular structures in the mineralized matrix [Figure 3(G)].

After calcium hydroxide application, a thick acellular necrotic zone was observed directly beneath the biomaterial and a connective tissue zone with a denser and homogenous tissue was seen directly underneath [Figure 4(A)].
While at 1 day, no mineralized foci formation was observed, after 14 and 28 days, the pulp tissue was characterized by the appearance of foci containing sequestered cells similar to those described with MTA. The size of the foci was heterogenous and their form was various: some were spherical while others were elongated and connected 

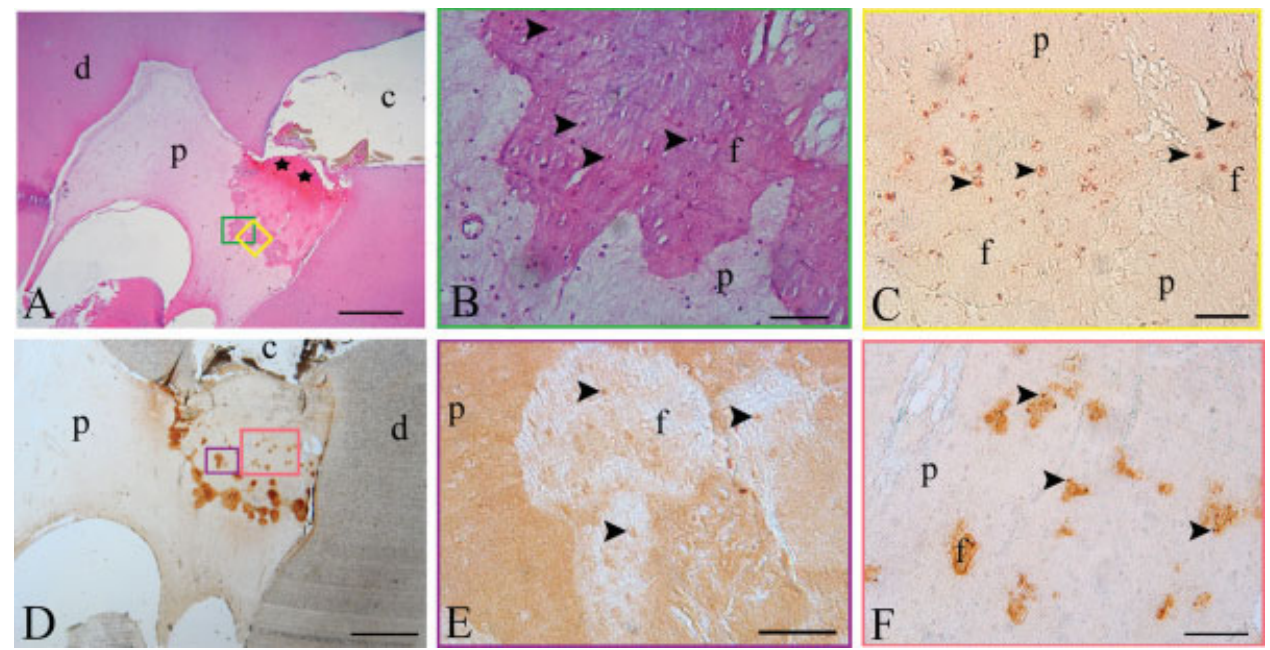

Figure 4. A view of pulp cusp area of a tooth capped with calcium hydroxide $X R^{\circledR}$ and cultured for 2 weeks after the BrdU labeling. A superficial thick acellular zone is observed directly beneath the calcium hydroxide (stars) and a connective tissue with a denser and homogenous tissue is seen directly underneath (A). This zone is delimited from the rest of pulp by mineralized foci containing sequestered cells (arrowheads) (A, B). The sequestered cells are BrdU-labelled (arrowheads) (C). Dentin sialoprotein immunolabeling is localized in pulp tissue and in the sequestered cells (arrowheads) $(E)$. Nestin is strongly expressed in the odontoblasts and in the mineralized foci sequestered cells (arrowheads) (D and F). A and B: H\&E staining. d, dentin; p, pulp; c, cavity; f, foci. Scale bars: $A$ and $\mathrm{D}=500 \mu \mathrm{m} ; \mathrm{E}=100 \mu \mathrm{m} ; \mathrm{B}, \mathrm{C}$, and $\mathrm{F}=50 \mu \mathrm{m}$. [Color figure can be viewed in the online issue, which is available at www.interscience.wiley.com.]

to each other [Figure $4(\mathrm{~A}, \mathrm{~B})]$. In some teeth, they completely sealed the exposure site surface [Figure 4(A)]. The cells sequestered in these foci were BrdU-labeled [Figure $4(\mathrm{C})]$.

The molecular characterization of the mineralized foci [Figure 4(D-F)] showed that Dentin sialoprotein immunolabeling was similar to collagen I expression. It was localized in the pulp matrix, mineralized foci matrix and in the sequestered cells [Figure 4(E)]. Nestin immunoreactivity was observed in the odontoblasts and in the mineralized foci. Its intensity was very strong in the sequestered cells [Figure 4(D,F)].

\section{Scanning Electron Microscopy}

SEM examination of the dentin and the mineralized structures [Figure 5] revealed the typical regular tubular appearance of dentin [Figure 5(A,C)] while irregular cavities of about $10 \mu \mathrm{m}$ in diameter and much smaller tubular-like structures with irregular diameters of $1-4 \mu \mathrm{m}$ were observed in the mineralized foci [Figure 5(B,D)]. No difference was observed between the mineralization obtained with calcium hydroxide and MTA.

\section{Mineralization Morphometric Analyses}

When teeth capped with MTA were compared to those capped with calcium hydroxide, ANOVA showed a statistically significant difference between both materials for the mineralization distance from the exposure $(p=0.001)$ while the comparison of pulp surface mineralization percentage was not statistically significant (Table IA).

When the mineralization was studied in function of the culture period using the combined data for both biomaterials, the ANOVA showed a statistically significant difference amongst the three culture periods for the pulp surface mineralization $(p=0.01)$ and the distance from the exposure site $(p=0.003)$. The Duncan test showed that there was a statistically significant increase in mineralization percentage from 1 to 14 days and a statistically significant decrease from 14 to 28 days. Similarly, the mineralization distance from the exposure site showed a statistically significant increase from 1 to 14 days and a statistically significant decrease from 14 to 28 days (Table IB).

\section{DISCUSSION}

This work demonstrates that the BrdU-labeled progenitor pulp cells can differentiate into odontoblast-like cells secreting a mineralized matrix. This mineralization was obtained with classically used capping materials and took the appearance of osteodentin which showed aspects of the morphological and molecular characteristics of reparative dentin. The pulpal response obtained with pulp capping materials here reproduces some features of the pulp reaction to these materials reported in vivo.

Direct pulp capping with calcium hydroxide induced mineralized foci formation in the pulp chamber after 2 and 4 weeks. These structures had various sizes and forms and were always facing the capping material. Although there 

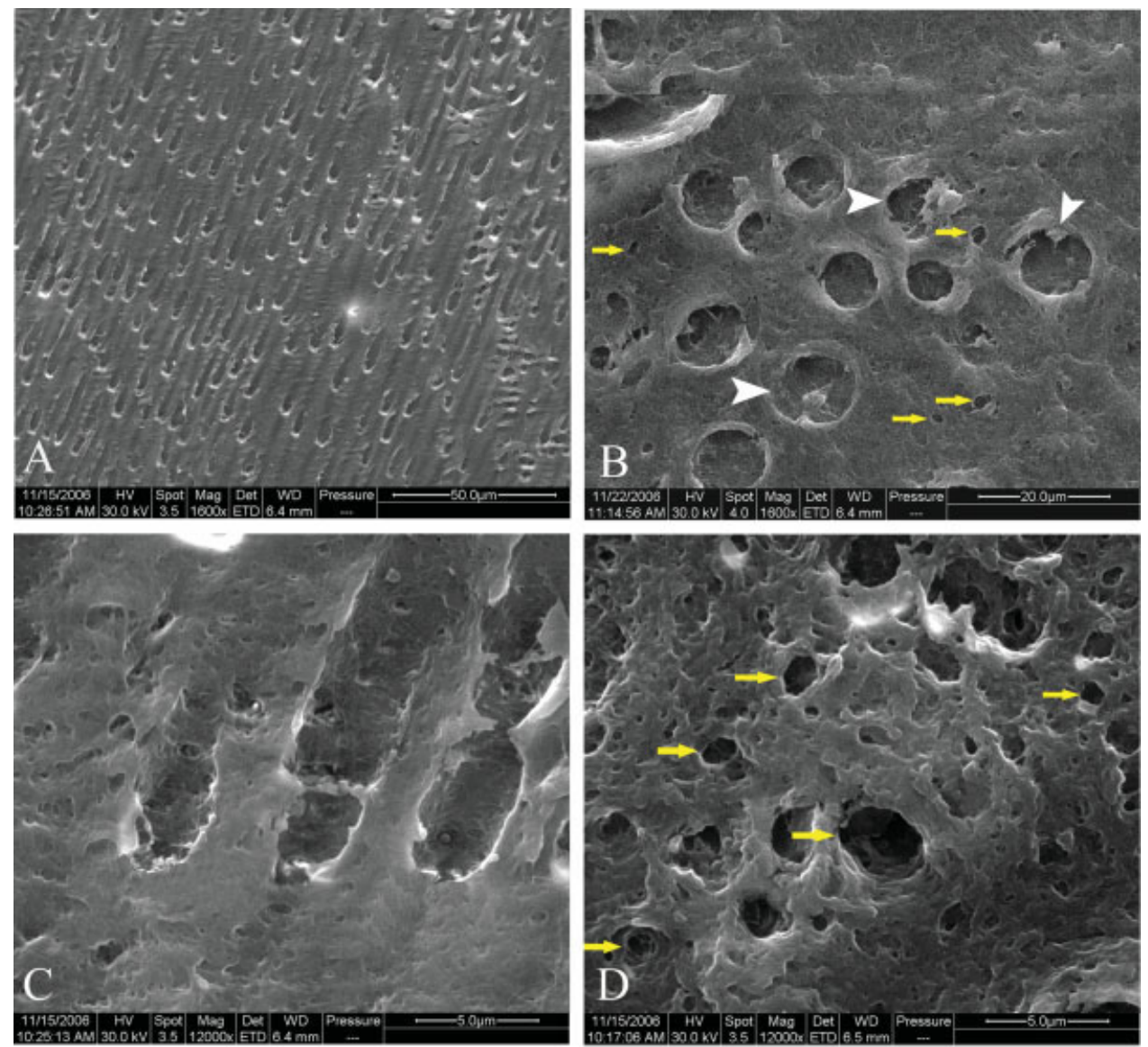

Figure 5. SEM view of dentin and mineralized foci after direct capping with calcium hydroxide ${ }^{\circledR}$ and culture for 2 weeks. Regular typical tubular structures can be observed in the dentin (A, C). Lacunae containing cells showing a larger diameter than dentin tubules can be seen in the mineralized deposition (arrowheads) (B). Smaller irregular tubular-like structures with diameters comparable to those of dentin tubules are also observed (arrows) $(B, D)$. Original magnifications: $A, B=\times 1600$; $C, D=\times 12,000$. [Color figure can be viewed in the online issue, which is available at www. interscience.wiley.com.]

was no statistically significant difference in the mineralization percentage between MTA and $\mathrm{Ca}(\mathrm{OH})_{2}$, the mineralized foci were located at a significant distance from the exposure site under $\mathrm{Ca}(\mathrm{OH})_{2}$. The strongly alkaline $\mathrm{pH}$ of $\mathrm{Ca}(\mathrm{OH})_{2}$ may contribute significantly to its action. Initially, this high $\mathrm{pH}$ appears to cause local necrosis of the pulp tissue around the injury site, and migration of pulp cells to this area is seen after $\sim 2$ weeks. ${ }^{8}$ A weaker chemical effect can be observed on the more apical tissue results in a necrotic zone which may stimulate the vital pulp tissue to respond by secreting the reparative dentin. ${ }^{9}$ This may explain the fact that the mineralization obtained with calcium hydroxide appeared after 2 weeks and was distant from the exposure site.

Pulpal response to MTA was different from that obtained with calcium hydroxide $\mathrm{XR}^{\circledR}$. A mineralized area appeared in the pulp tissue in the vicinity of the exposure after the first day of culture and significantly increased in size after 2 and 4 weeks. The early mineralization obtained here after BrdU-labeling for 1 day and tooth culture for an additional day is surprising. To our knowledge, the mineralization after such a delay has never been studied in vivo. However, the activation and presence of progenitor BrdUlabeled cells have been shown in vitro in close association with the exposure site after 1 day. ${ }^{7}$ The significance of such early mineralization remains to be determined, but this early mineralization may be partially explained by the reported MTA biocompatibility ${ }^{10}$ and calcium release. ${ }^{11}$ The mineralization with MTA was more localized, homogenous, thin, and very close to the exposure site as compared to that obtained with calcium hydroxide. These results corroborate those observed in a comparative study between these two materials in vivo which have shown that dentin bridge formation with MTA was more homogenous and more localized than that formed with calcium hydroxide. $^{12}$

The mineralization obtained in this work took the form of osteodentin. This form of mineralization may precede the dentin bridge formation observed after $\mathrm{Ca}(\mathrm{OH})_{2}$ and MTA application. Seven days after human teeth capping 


\begin{tabular}{llr}
\hline & $\begin{array}{c}\text { Pulp Surface } \\
\text { Mineralization } \\
\text { Percentage: } \\
\text { Mean (SD) }\end{array}$ & $\begin{array}{r}\text { Distance from } \\
\text { Exposure in } \mu \mathrm{m}: \\
\text { Mean (SD) }\end{array}$ \\
\hline A. Product & & \\
$\mathrm{MTA}$ & $0.58(0.66)$ & $172(107)$ \\
$\mathrm{CaOH}_{2}$ & $1.34(1.54)$ & $715(654)$ \\
B. Culture period (days) & & $55(103)^{\mathrm{a}}$ \\
1 & $0.07(0.13)^{\mathrm{a}}$ & $1058(745)^{\mathrm{b}}$ \\
14 & $2.47(1.6)^{\mathrm{b}}$ & $358(309)^{\mathrm{c}}$ \\
28 & $0.64(0.64)^{\mathrm{c}}$ & \\
\hline
\end{tabular}

These were performed on hematoxylin-eosin stained sections to compare the mineralization obtained in function of the material: MTA or $\mathrm{Ca}(\mathrm{OH})_{2}(\mathrm{~A})$ and the culture period using the combined data for both materials (B). The pulp surface mineralization percentage was calculated from the pulp chamber surface and the total foci area.

ANOVA showed a statistically significant difference between both materials regarding the distance from exposure site $(p=0.001)$ but not the mineralization percentage (A). The same test showed a statistically significant difference in function of the culture period $(p=0.01)$. Duncan test showed a statistically significant increase in the mineralization percentage and distance from exposure site from one to 14 days and a decrease from 14 to 28 days (B). Groups identified with superscript letters indicate statistically significant groups.

with calcium hydroxide, electron-dense mineral deposits associated with collagen fibrils were observed. ${ }^{13}$ Similarly, after pulp capping for 2 weeks in dogs with MTA, irregular osteotypic matrix depositions were observed ${ }^{14}$ while the reparative tubular dentin bridge formation with this material in human teeth was obtained after 2 months. ${ }^{12}$

Thus, the mineralization obtained in this work corresponds to one of the early steps of the reparative dentin processes reported in vivo and appears after an equivalent delay.

It is unclear from in vivo studies what this osteotypic matrix becomes after later periods. The only thing which is often reported in the literature is that it is often followed by another form of reparative dentin. Surprisingly, in this work we report a significant decrease of the mineralized foci surface with both materials from 2 to 4 weeks. Although our data cannot explain the mechanism or the significance of this observation, to our knowledge, this is the first report of such a decrease.

The signaling processes responsible for MTA and $\mathrm{Ca}(\mathrm{OH})_{2}$-induced odontoblast-like cell differentiation are beyond the scope of this study. However, it has been suggested that alterations in calcium levels in the cellular environment can invoke responses of apoptosis or differentiation of odontoblast-like cells. ${ }^{15}$ Accordingly, calcium is the principal component in both capping agents and may be involved in pulp cells differentiation and it has been shown that human dental pulp cells cultured on $\mathrm{Ca}(\mathrm{OH})_{2}$ differentiate into odontoblast-like cells after 4 weeks, synthesize type I but not type III collagen, and display apical accumulation of actin and vimentin. ${ }^{16} \mathrm{~A}$ recent study has demonstrated that calcium hydroxide may exert its effects on dentin regeneration by initiating the release of dentin matrix-bound bioactive molecules particularly TGF$\beta 1$ to signal reparative dentinogenesis. ${ }^{17}$

The osteodentin decribed in vivo has been so far qualified as nonspecific but said to play a role in initiating reparative dentinogenesis. ${ }^{18}$ However, this description was always based on morphological criteria.

Under pathological conditions such as trauma or severe carious lesions, reparative dentinogenesis requires cell proliferation, migration, and differentiation of progenitor cells into odontoblast-like cells to secrete reparative dentin to isolate the subjacent pulp from the injury site and/or the pulp capping material. Accordingly, although this may appear as coincidental in this work, the mineralized matrix seem to be secreted by newly differentiated progenitor cells as the sequestered cells in the mineralized foci under both capping materials were BrdU-labeled. Immunochemistry revealed that the molecular markers of dentin were expressed in the mineralized foci matrix where irregular tubular structures characteristic of dentin were observed. Moreover, the molecular markers of the odontoblasts such as collagen I, dentin sialoprotein, and nestin were expressed at a high level in the sequestered cells. Nestin expression in the sequestered cells is very significant as the expression of this intermediate filament protein have been shown as specific and characteristic of the secretory human odontoblast. ${ }^{19}$ Thus, the expression of these proteins together with nestin in these cells strongly suggests that these cells are odontoblastic and the mineralized foci seem to correspond to a form of reparative dentin production rather than a nonspecific mineral deposition.

Reparative dentin production has been reported in vivo and only on animal models in vitro. ${ }^{20}$ Culture techniques were developed to study reparative dentinogenesis using rodent and human dentin slices. ${ }^{21,22}$ While interspecies differences should be considered with the rodent tooth slice culture method, it is difficult to distinguish the responses to pulpal from dentin cavities in the human slice culture method as in both cases a very large pulpal injury is performed in order to prepare the dentin slices. ${ }^{21,22}$

Our previous work have shown that perivascular progenitor pulp cells in human teeth can be activated and migrate to the injury site after pulpal injury. This work provides direct evidence that cells can differentiate and secrete a form of reparative dentin in entire human tooth in vitro. It has to be mentioned that this culture model has some limitations. There is a lack of $\mathrm{CO}_{2}$ and $\mathrm{O}_{2}$ exchange and the cells may be stressed by a lower $\mathrm{pH}$ which may influence the overall response to stimuli and the entire inflammatory response and clot resolution cannot be simulated. However, this model reproduces the clinical situation for pulpal cavities preparations. Although its value in studying reparative dentinogenesis requires further investigation, this model may be useful in predicting the biocompatibility of dental materials.

We thank Dr. Jean-Charles Gardon for providing the third molars and Dr. W. T. Butler for the anti-dentin sialoprotein antibodies. 


\section{REFERENCES}

1. Linde A, Goldberg M. Dentinogenesis. Crit Rev Oral Biol Med 1993;4:679-728.

2. Smith AJ, Lesot H. Induction and regulation of crown dentinogenesis: Embryonic events as a template for dental tissue repair? Crit Rev Oral Biol Med 2001;12:425-437.

3. Smith AJ. Pulpal responses to caries and dental repair. Caries Res 2002;36:223-232.

4. Tziafas D. Basic mechanisms of cytodifferentiation and dentinogenesis during dental pulp repair. Int J Dev Biol 1995;39: 281-290.

5. Gronthos S, Brahim J, Li W, Fisher LW, Cherman N, Boyde A, DenBesten P, Robey PG, Shi S. Stem cell properties of human dental pulp stem cells. J Dent Res 2002;81:531-535.

6. About I, Bottero MJ, de Denato P, Camps J, Franquin JC, Mitsiadis TA. Human dentin production in vitro. Exp Cell Res 2000;258:33-41.

7. Tecles O, Laurent P, Zygouritsas S, Burger AS, Camps J, Dejou J, About I. Activation of human dental pulp progenitor/ stem cells in response to odontoblast injury. Arch Oral Biol 2005;50:103-108.

8. Schroder U. Effects of calcium hydroxide-containing pulpcapping agents on pulp cell migration, proliferation, and differentiation. J Dent Res 1985;64 (Spec No.):541-548.

9. Stanley HR. Pulp capping: Conserving the dental pulp-Can it be done? Is it worth it? Oral Surg Oral Med Oral Pathol 1989;68:628-639.

10. Camilleri J, Pitt Ford TR. Mineral trioxide aggregate: A review of the constituents and biological properties of the material. Int Endod J 2006;39:747-754.

11. Duarte MA, Demarchi AC, Yamashita JC, Kuga MC, Fraga Sde C. $\mathrm{pH}$ and calcium ion release of 2 root-end filling materials. Oral Surg Oral Med Oral Pathol Oral Radiol Endod 2003;95:345-347.

12. Aeinehchi M, Eslami B, Ghanbariha M, Saffar AS. Mineral trioxide aggregate (MTA) and calcium hydroxide as pulp-cap- ping agents in human teeth: A preliminary report. Int Endod J 2003;36:225-231.

13. Yoshiba K, Yoshiba N, Nakamura H, Iwaku M, Ozawa H. Immunolocalization of fibronectin during reparative dentinogenesis in human teeth after pulp capping with calcium hydroxide. J Dent Res 1996;75:1590-1597.

14. Tziafas D, Pantelidou O, Alvanou A, Belibasakis G, Papadimitriou S. The dentinogenic effect of mineral trioxide aggregate (MTA) in short-term capping experiments. Int Endod J 2002;35:245-254.

15. Kardos TB, Hunter AR, Hanlin SM, Kirk EE. Odontoblast differentiation: A response to environmental calcium? Endod Dent Traumatol 1998;14:105-111.

16. Seux D, Couble ML, Hartmann DJ, Gauthier JP, Magloire H. Odontoblast-like cytodifferentiation of human dental pulp cells in vitro in the presence of a calcium hydroxide-containing cement. Arch Oral Biol 1991;36:117-128.

17. Graham L, Cooper PR, Cassidy N, Nor JE, Sloan AJ, Smith AJ. The effect of calcium hydroxide on solubilisation of bioactive dentine matrix components. Biomaterials 2006;27: 2865-2873.

18. Braut A, Kollar EJ, Mina M. Analysis of the odontogenic and osteogenic potentials of dental pulp in vivo using a Col1a12.3-GFP transgene. Int J Dev Biol 2003;47:281-292.

19. About I, Laurent-Maquin D, Lendahl U, Mitsiadis TA. Nestin expression in embryonic and adult human teeth under normal and pathological conditions. Am J Pathol 2000;157:287-295.

20. Sloan AJ, Smith AJ. Stimulation of the dentine-pulp complex of rat incisor teeth by transforming growth factor-beta isoforms 1-3 in vitro. Arch Oral Biol 1999;44:149-156.

21. Smith AJ, Tobias RS, Cassidy N, Plant CG, Browne RM, Begue-Kirn C, Ruch JV, Lesot H. Odontoblast stimulation in ferrets by dentine matrix components. Arch Oral Biol 1994;39:13-22.

22. Magloire H, Joffre A, Bleicher F. An in vitro model of human dental pulp repair. J Dent Res 1996;75:1971-1978. 